University of Massachusetts Amherst

ScholarWorks@UMass Amherst

1998

\title{
Effect of high salt concentrations on the determination of arsenic and selenium by flow injection hydride generation electrothermal atomic absorption spectrometry
}

Robert I. Ellis

University of Massachusetts Amherst

Nils G. Sundin

University of Massachusetts Amherst

Julian Tyson

University of Massachusetts Amherst

Susan A. McIntosh

Christopher P. Hanna

See next page for additional authors

Follow this and additional works at: https://scholarworks.umass.edu/chem_faculty_pubs

Part of the Chemistry Commons

\section{Recommended Citation}

Ellis, Robert I.; Sundin, Nils G.; Tyson, Julian; McIntosh, Susan A.; Hanna, Christopher P.; and Carnrick, Glen, "Effect of high salt concentrations on the determination of arsenic and selenium by flow injection hydride generation electrothermal atomic absorption spectrometry" (1998). Analyst. 1422.

Retrieved from https://scholarworks.umass.edu/chem_faculty_pubs/1422 
Authors

Robert I. Ellis, Nils G. Sundin, Julian Tyson, Susan A. McIntosh, Christopher P. Hanna, and Glen Carnrick 


\title{
Effect of high salt concentrations on the determination of arsenic and selenium by flow injection hydride generation electrothermal atomic absorption spectrometry
}

\author{
Robert I. Ellis ${ }^{a}$, Nils G. Sundin ${ }^{a}$, Julian F. Tyson ${ }^{*}$, Susan A. McIntosh ${ }^{b}$, Christopher P. \\ Hanna $^{b}$ and Glen Carnrick ${ }^{b}$ \\ a Department of Chemistry, University of Massachusetts, Box 34510, Amherst, MA 01003-4150, \\ USA \\ b Perkin-Elmer Corporation, 761 Main Avenue, Norwalk, CT 06897, USA
}

In the determination of arsenic and selenium by flow injection hydride generation ETAAS, the presence of up to $20 \%$ sodium chloride enhanced the signals for 20 $\mu \mathrm{g} \mathrm{I}^{-1}$ arsenic and selenium by up to $28 \%$. The enhancement was obtained with a variety of gas-liquid separators. A systematic study of the possible causes of the signal enhancement in the determination of selenium was undertaken, from which it was concluded that the effect originated in the processes responsible for the distribution of the hydrogen selenide between the solution and gas phases. Processes related to the transport of the analyte from the gas-liquid separator and the trapping of the analyte on the interior of the atomizer were not affected by the presence of dissolved salts. As sodium was found to be transported to the atomizer, it was deduced that aqueous aerosol was deposited in the atomizer, although the quantities were irreproducible. The enhancement could be eliminated by increasing the borohydride concentration. However, with the small volume gas-liquid separator, this latter approach was limited because of carry-over of liquid to the atomizer. The effect could be compensated for by adding up to $40 \%$ $\mathbf{m} / \mathbf{v}$ of salt to the borohydride reagent.

Keywords: Flow injection; hydride generation;

electrothermal atomic absorption spectrometry; selenium; arsenic; electrolytes; salting-out

Flow injection hydride generation electrothermal atomic absorption spectrometry (FI-HG-ETAAS) is an emerging technique for the determination of several trace elements, including selenium and arsenic. ${ }^{1,2}$ The procedure combines the high precision and sample throughput of flow injection, the ability to separate the analyte from the matrix by hydride generation, and the high sensitivity and low detection limit of ETAAS. A discrete volume of acidified sample solution is merged with sodium borohydride solution, and the volatile hydride of the trace element which is formed is transferred into the gas phase by (a) the evolution of hydrogen from the decomposition of the excess borohydride and (b) the merging of an argon stream. Following bulk gas-liquid separation, the hydride is trapped on the interior surface of the graphite tube atomizer of an electrothermal atomic absorption spectrometer, which has been pre-treated with a trapping agent (e.g., iridium chloride solution) prior to atomization.

A possible area of application of this methodology is in the analysis of solutions containing high concentrations of dissolved salts, such as sea-waters, industrial brines and digests of solids with predominantly inorganic matrices such as sludges and sediments. Initial studies ${ }^{3}$ of the determination of selenium in solutions containing up to $20 \%$ sodium chloride by FI-HGETAAS indicated that the presence of salt caused signal enhancements (up to 127\%). To our knowledge, this effect has not been reported previously.

In this paper, the results of further studies of this effect are presented. The high recoveries are confirmed and a number of possible causes are proposed. The results of a systematic investigation of these possible causes are discussed, from which it is concluded that the diminished solubility of gases in the presence of dissolved salts (the so-called 'salting-out' effect) was responsible. Two possible procedures for overcoming this interference were investigated.

\section{Experimental}

Reagents and supplies

Working standard $\mathrm{Se}^{\mathrm{IV}}$ and $\mathrm{As}{ }^{\mathrm{V}}$ solutions were made by diluting an aliquot of the respective stock standard solution (PerkinElmer, Norwalk, CT, USA) to produce a concentration of 1000 $\mu \mathrm{g}^{-1}$. The carrier stream was $10 \% \mathrm{v} / \mathrm{v}$ hydrochloric acid (Fisher, Pittsburgh, PA, USA) and the reductant stream was $0.2 \% \mathrm{~m} / \mathrm{v} \mathrm{NaBH}_{4}$ (Fisher) prepared daily and stabilized with $0.05 \% \mathrm{~m} / \mathrm{v} \mathrm{NaOH}$ (Fisher). Samples containing selenium were prepared by placing $10 \mathrm{ml}$ of $10 \% \mathrm{v} / \mathrm{v} \mathrm{HCl}$ in a $100 \mathrm{ml}$ calibrated flask followed by the appropriate amount of salt where required. Approximately $30 \mathrm{ml}$ of purified water were added before adding an aliquot of selenium standard solution and dilution to volume. Samples containing arsenic were prepared by placing $10 \mathrm{ml}$ of $10 \% \mathrm{v} / \mathrm{v} \mathrm{HCl}, 10 \mathrm{ml}$ of $1 \% \mathrm{~m} / \mathrm{v} \mathrm{KI}$ (Fisher) and $10 \mathrm{ml}$ of $1 \% \mathrm{~m} / \mathrm{v}$ ascorbic acid (Fisher) in a calibrated flask followed by addition of an aliquot of arsenic standard solution. These samples were stoppered and left at room temperature for $1 \mathrm{~h}$ to allow reduction of $\mathrm{As}^{\mathrm{V}}$ to $\mathrm{As} \mathrm{s}^{\mathrm{III}}$ prior to addition of salt when required and dilution to volume with high purity water.

In the investigation into the cause of the increased signals, solutions of $\mathrm{Se}^{\mathrm{IV}}$, prepared as above, of concentrations from 0 to $20 \mu \mathrm{g}^{-1}$ containing either 0 or $20 \%$ of sodium chloride were used unless specified otherwise.

\section{Instrumentation}

A multi-line flow injection manifold ${ }^{4}$ was used in which the sample was injected into an acid carrier, then merged with the alkaline borohydride reagent. After passage through a reaction coil, the reaction zone was merged with an argon stream, passed through a stripping coil and delivered to the gas-liquid separator. Drainage from the gas-liquid separator was controlled by a peristaltic pump channel. The manifold was used in conjunction with a Perkin-Elmer Model 4100ZL Zeeman corrected electrothermal atomic absorption spectrometer interfaced with a Digital 316sx workstation, and controlled using 
Perkin-Elmer Gem software (version 7.2.1). The manifold was constructed from PTFE manifold tubing (1 mm id) and a PerkinElmer Chemifold and gas-liquid separator. The carrier stream flow rate was $4 \mathrm{ml} \mathrm{min}^{-1}$ and that of the borohydride reagent was $6 \mathrm{ml} \mathrm{min}-1$. The waste line from the gas-liquid separator could be pumped at up to $15 \mathrm{ml} \mathrm{min}-1$. The length of tubing between the confluence point and the addition of argon was 110 $\mathrm{cm}$ and the length between this point and the gas-liquid separator was $300 \mathrm{~cm}$. A Permapure Nafion dryer was fitted to the gas transfer line which connected the gas-liquid separator to the autosampler probe of the spectrometer. This removed moisture from the transferred gases. ${ }^{4}$ The FIAS program is given in Table 1 . The sample loop volume was $500 \mu$ l. The argon stripping gas flow rate was $130 \mathrm{ml} \mathrm{min}^{-1}$. The hydrides were trapped on a transversely heated graphite tube pre-treated with $120 \mu \mathrm{l}$ of $0.1 \% \mathrm{~m} / \mathrm{v}$ iridium chloride solution (PerkinElmer). ${ }^{5}$ Tubes which are pre-treated with iridium may be used for up to 300 firings provided that the temperature does not exceed $2300{ }^{\circ}$ C. 6 Perkin-Elmer System II electrodeless discharge lamps, operated at $260 \mathrm{~mA}$ (selenium) and $350 \mathrm{~mA}$ (arsenic), were used, with detection at a wavelength of $196.0 \mathrm{~nm}$ for selenium and $193.7 \mathrm{~nm}$ for arsenic. Peaks were quantified by area. The furnace programs are given in Table 2. Experiments with quartz tube atomization were performed with a PerkinElmer Model 3100 atomic absorption spectrometer fitted with a quartz tube atomizer positioned within an air-acetylene flame and a Perkin-Elmer selenium hollow cathode lamp operated at $15 \mathrm{~mA}$. All other relevant parameters were as described above. Two other gas-liquid separators were studied; one was supplied by PS Analytical (Orpington, Kent, UK) for use in a continuous flow hydride generator and the second was constructed in-house from borosilicate glass and is shown schematically in Fig. 1. The use of the first of these devices has been described numerous times (see, for example, refs. 8, 10 and 11).

\section{Procedures}

Effect of salts on signal

Solutions of $\mathrm{Se}^{\mathrm{IV}}\left(1-10 \mu \mathrm{g} 1^{-1}\right)$ and $\mathrm{As}^{\mathrm{III}}\left(5\right.$ and $\left.10 \mu \mathrm{g} 1^{-1}\right)$ were prepared as described above, either with or without salts, to

\begin{tabular}{|c|c|c|c|c|c|}
\hline Step & Time/s & Pump 1/rpm & Pump 2/rpm & Valve & Comments \\
\hline Pre-fill & 10 & 100 & 0 & Fill & $\begin{array}{l}\text { Fill loop and pre- } \\
\text { heat atomizer }\end{array}$ \\
\hline 1 & 20 & 100 & 80 & Fill & Fill loop \\
\hline 2 & 8 & 0 & 0 & Inject & $\begin{array}{l}\text { Insert probe to } \\
\text { atomizer }\end{array}$ \\
\hline 3 & 30 & 0 & 80 & Inject & $\begin{array}{l}\text { Generate hydride } \\
\text { and trap }\end{array}$ \\
\hline 4 & 8 & 0 & 0 & Inject & $\begin{array}{l}\text { Remove probe } \\
\text { from atomizer }\end{array}$ \\
\hline 5 & 5 & 0 & 80 & Fill & Begin atomization \\
\hline
\end{tabular}

Table 2 Furnace parameters

\begin{tabular}{lcccccc}
$\begin{array}{l}\text { Ele- } \\
\text { ment }\end{array}$ & Step & $\begin{array}{c}\text { Tempera- } \\
\text { ture }{ }^{\circ} \mathrm{C}\end{array}$ & Ramp & $\begin{array}{c}\text { Gold/s } / \mathrm{s} \text { How } \\
\text { rate/ml } \\
\mathrm{min}^{-1}\end{array}$ & Read/s \\
Se & Hold & 250 & 1 & 15 & 250 & \\
& Atomize & 2000 & 0 & 5 & 0 & 5 \\
As & Clean & 2300 & 1 & 3 & 250 & \\
& Hold & 400 & 1 & 15 & 250 & \\
& Atomize & 2100 & 0 & 5 & 0 & 5 \\
& Clean & 2300 & 1 & 3 & 250 & \\
\hline
\end{tabular}

determine the percentage increase in signal compared with that obtained when no salt was present.

It was considered that the most plausible explanations for the effect were either an increase in (a) the efficiency of trapping of the hydride within the graphite tube (due to the presence of salt transported as aerosol), (b) the efficiency of the gas-liquid separation behaviour of the manifold components in the presence of salt or (c) the extent of formation of the hydride.

\section{Contamination}

Blank solutions containing salt were analyzed to ensure that the increase in signal did not result from contamination of the salts by trace concentrations of selenium or arsenic.

\section{Trapping efficiency}

It is possible that some of the dissolved salt may be transferred to the graphite tube in the form of an aerosol. Several workers have speculated that this occurs, ${ }^{7-9}$ but it has been conclusively demonstrated recently by Wickstrom et al. ${ }^{10}$ for a continuousflow hydride generation system with a Thompson-type ${ }^{11}$ gasliquid separator. If the trapping efficiency is below $100 \%$, there may be an improvement in trapping efficiency in the presence of the transferred salt aerosol. The trapping temperature of the furnace was varied between 20 and $800{ }^{\circ} \mathrm{C}$ and untreated graphite tubes or tubes treated with Pd were also used.

A sodium hollow cathode lamp was fitted to the spectrometer and the amount of sodium in the graphite tube accumulated during the hydride generation of selenium from solutions with and without sodium chloride was measured.

A blank solution containing $20 \% \mathrm{~m} / \mathrm{v}$ of sodium chloride was injected into the manifold and carried through the procedure, omitting the furnace firing stage. The furnace was held at the trapping temperature and a sample without salt was injected. The furnace was then fired in the normal manner. If salt in the furnace had the effect of increasing the trapping efficiency, there would be an increase in signal in the latter determination.

To determine whether the effect was exclusive to electrothermal atomization, solutions of between 0 and $30 \mu \mathrm{g} 1^{-1} \mathrm{Se}$, containing either 0 or $20 \% \mathrm{~m} / \mathrm{v}$ of $\mathrm{NaCl}$, were analyzed using quartz tube atomization.

\section{Kinetic effects}

An increase in the rate of the hydride forming reaction in the presence of dissolved salts could be responsible for the enhancement. If this was the case, increasing the length of the manifold tubing would allow a longer time for reaction and hence could eliminate the increase in signal. To investigate the effect of changing manifold parameters, a systematic study which involved varying the length of manifold tubing was carried out. The effect of including a stopped-flow period was also studied.

\section{Transfer between gas-liquid separator and atomizer}

To investigate whether a greater proportion of the analyte was being transferred to the graphite tube by an aerosol formed in

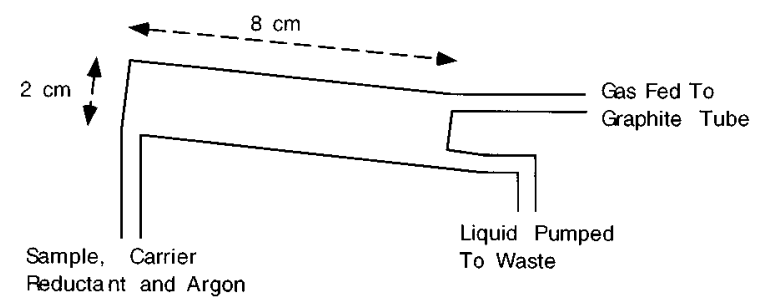

Fig. 1 'Spray chamber' design of gas-liquid separator, constructed inhouse. 
the presence of the salt, the Nafion dryer was removed from the gas transfer line and replaced with either a PTFE tube or a drying tube containing magnesium perchlorate. The composition of aerosol generated from concentrated salt solutions can be different from that generated from low ionic strength solutions, an effect known as aerosol ionic redistribution. ${ }^{12}$

\section{Batch versus flow}

A batch system was constructed as shown in Fig. 2 to check whether the increase in signal was specific to flow injection systems.

\section{Gas-liquid separation}

To investigate the efficiency of gas-liquid separation, a second gas-liquid separator was coupled to the waste line from the first gas-liquid separator as shown in Fig. 3. Should there be an increase in efficiency in the presence of salt, there would be a lower signal from the second gas-liquid separator compared with that obtained in the absence of salt. The signals for both a blank and a standard in the presence and absence of $20 \%$ of sodium chloride were obtained for the other two gas-liquid separator devices. The device from PS Analytical was operated with an additional hydrostatic head on the waste in order to compensate for the higher back-pressure of the narrow tubing connecting the device to the graphite furnace atomizer than the normal back-pressure from a quartz tube atomizer and associated connecting tubing. The optimized argon flow rate was $150 \mathrm{ml} \mathrm{min}-1$. The 'spray chamber' device was also operated at an optimized gas flow rate of $150 \mathrm{ml} \mathrm{min}^{-1}$.

\section{Overcoming the effect}

Because the increase in signal in the presence of high electrolyte concentrations would require matrix matching of standards to obtain accurate analyses, two ways to overcome the problem were investigated: variation of the borohydride concentration and the addition of salt to the reagents. The effect of changing the borohydride concentration over the range $0.2-2.0 \%$ was investigated. At high concentrations of sodium borohydride, foaming in the gas-liquid separator may cause liquid to rise

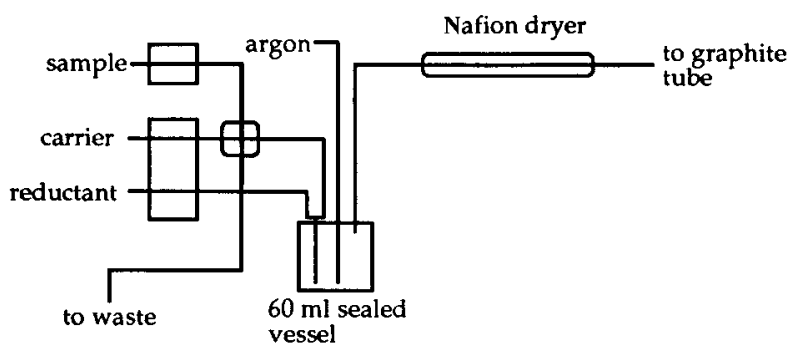

Fig. 2 Batch hydride generation manifold.

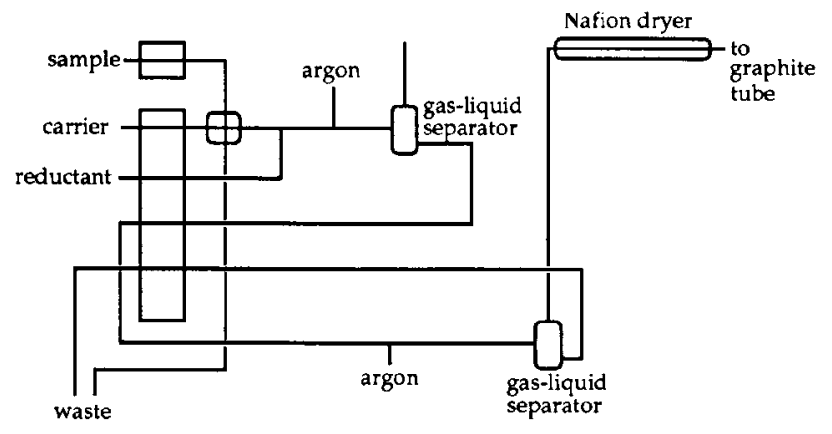

Fig. 3 Flow injection manifold with second gas-liquid separator. through the gas transfer tube and be deposited on the furnace, thereby impeding the trapping ability. Therefore, a second waste line was fitted to the gas-liquid separator and a $10 \mathrm{~mm} \times$ $8 \mathrm{~mm}$ id acrylic tube was inserted into the gas transfer line to prevent liquid droplets from reaching the graphite tube. The tube was used in place of the Nafion dryer and was removed and thoroughly cleaned between measurements. Salt was added to the sample in the manifold from an extra line which merged with the sample carrier stream upstream of the merging with borohydride. The effect of adding salt to the borohydride reagent was also investigated.

\section{Results and discussion}

\section{Effect of salts on signal enhancement}

Typical recoveries for solutions of $\mathrm{As}^{\mathrm{III}}$ and $\mathrm{Se}^{\mathrm{IV}}$ are given in Table 3. The effect was observed for both arsenic and selenium in the presence of sodium chloride but was most pronounced in the determination of selenium in the presence of potassium sulfate.

\section{Contamination}

There was no measurable amount of selenium or arsenic in any of the salts used.

\section{Trapping efficiency}

Although sodium was observed in the furnace in amounts in excess of the background, leading to the conclusion that aerosol droplets are transported to the furnace, the sodium signals were highly irreproducible. As the increase in signal in the presence of sodium chloride is precise, it is unlikely that the variable amounts of sodium on the furnace would be a part of the mechanism responsible for this effect. High signals were not observed for a solution run subsequently to a high-salt blank for which the furnace had not been fired. It was concluded that the increased signals in the presence of salt were not due to processes inside the graphite tube, such as increased trapping efficiency.

For the analyses with quartz tube atomization, signal enhancements of between 121 and $127 \%$ were obtained for the analysis of the solutions containing $30 \mu \mathrm{g} \mathrm{l}^{-1} \mathrm{Se}$ and $20 \% \mathrm{~m} / \mathrm{v}$ of $\mathrm{NaCl}$. It is therefore unlikely that the increase is specific to graphite tube atomization.

Table 3 Typical recovery data

\begin{tabular}{|c|c|c|c|}
\hline Analyte & Salt & $\begin{array}{c}\text { Salt concentration } \\
(\%)\end{array}$ & $\begin{array}{l}\text { Signal* relative to } \\
\text { that for a solution } \\
\text { with no salt }(\%)\end{array}$ \\
\hline \multirow[t]{11}{*}{$\operatorname{Se}\left(5 \mathrm{ng} \mathrm{ml}^{-1}\right)$} & $\mathrm{NaCl}$ & 0.62 & $107 \pm 4$ \\
\hline & & 1.25 & $109 \pm 4$ \\
\hline & & 2.5 & $112 \pm 4$ \\
\hline & & 5 & $115 \pm 4$ \\
\hline & & 10 & $120 \pm 5$ \\
\hline & & 20 & $126 \pm 5$ \\
\hline & $\mathrm{K}_{2} \mathrm{SO}_{4}$ & 0.31 & $104 \pm 4$ \\
\hline & & 0.62 & $108 \pm 4$ \\
\hline & & 1.25 & $110 \pm 4$ \\
\hline & & 2.5 & $112 \pm 4$ \\
\hline & & 5 & $112 \pm 4$ \\
\hline \multirow[t]{2}{*}{ As $\left(5 \mathrm{ng} \mathrm{ml}^{-1}\right)$} & $\mathrm{NaCl}$ & 20 & $128 \pm 5$ \\
\hline & $\mathrm{K}_{2} \mathrm{SO}_{4}$ & 5 & $104 \pm 3$ \\
\hline
\end{tabular}




\section{Kinetic effects}

Neither lengthening the manifold nor incorporating a stoppedflow stage into the FIAS program affected the signal enhancement, although the sensitivity was affected. This suggests that the kinetics of the reaction are not significantly influenced by the presence of dissolved salt.

\section{Transfer between gas-liquid separator and atomizer}

When the Nafion dryer was replaced with either a PTFE tube or a magnesium perchlorate drying tube, the enhancement was unaffected, although the magnesium perchlorate drying tube reduced the sensitivity. This suggests that even if aerosol is transported through the gas transfer line, the transport of the analyte is unaffected by the presence of aerosol.

\section{Batch versus flow}

The batch experiment using the apparatus shown schematically in Fig. 2 did not result in a higher signal in the presence of dissolved salt, so the phenomenon was considered to be related to the flow procedure. The increase in signal in the presence of dissolved salt with the FI system was therefore due to greater transfer of the hydride to the vapor phase.

\section{Gas-liquid separation}

In the presence of salt, the signal for the selenium from the second gas-liquid separator (see Fig. 3) was $75 \%$ of that for solutions which did not contain salt, indicating that less of the analyte was pumped out of the first gas-liquid separator (to waste) in the presence of salt. That is, there was an increase in the proportion of the analyte which was transferred into the gas phase in the presence of dissolved salts. The performance of the various separator devices is shown in Table 4, from which it is clear that the effect is not a unique feature of the Perkin-Elmer device. Manifolds incorporating the other two devices also produced enhanced signals in the presence of salt, although both gave reduced peak area in comparison with peaks obtained with the Perkin-Elmer device, which is designed to maximize sensitivity by minimizing the dispersion in the headspace.

High concentrations of electrolytes in aqueous solution lower the solubility of dissolved gases in that solution. This effect is known as 'salting-out'..$^{13-15}$ The extent of the decrease in gas solubility is increased when both the charge on and size of the ions are increased, and with increasing ionic concentration. ${ }^{16}$ This phenomenon is commonly utilized in headspace sampling in gas chromatography. ${ }^{17}$ Salts are added to sample solutions in order to decrease the solubility of gases and vapors in solution and increase the partial pressure of the analyte species in the headspace.

In a FI system the process of formation of the hydride and removal from solution are initiated once the acidified sample and borohydride stream mix, as hydrogen gas (produced by the decomposition of excess borohydride) forms a bulk gas phase in contact with the bulk liquid phase. The process of removal of

Table 4 Performances of different gas-liquid separator devices

\begin{tabular}{|c|c|c|c|c|}
\hline \multirow[b]{2}{*}{ Device } & \multicolumn{4}{|c|}{ Integrated absorbance for selenium/s } \\
\hline & Blank, no salt & $\begin{array}{c}\text { Blank, with } \\
\text { salt }^{*}\end{array}$ & $\begin{array}{c}10 \mathrm{ng} \mathrm{ml}^{-1} \\
\text { no salt }\end{array}$ & $\begin{array}{l}10 \mathrm{ng} \mathrm{ml}{ }^{-1} \\
\text { with salt* }\end{array}$ \\
\hline Perkin-Elmer & 0.007 & 0.006 & 0.446 & 0.589 \\
\hline PS Analytical & 0.003 & 0.002 & 0.204 & 0.385 \\
\hline In-house & 0.007 & 0.007 & 0.259 & 0.499 \\
\hline
\end{tabular}

the hydride from the liquid phase continues with the addition of a stream of argon gas which changes the ratio of gas to liquid in the flowing stream. It is possible that further transfer occurs in the gas-liquid separator, the role of which is primarily that of separating bulk phases. Hydrogen selenide, unlike some other hydrides (including arsine), has a solubility in aqueous solution which can involve the formation of the corresponding anions $\left(\mathrm{Se}^{2-}\right.$ and $\left.\mathrm{HSe}^{-}\right)$and hence the acidity of the solutions is a factor which affects solubility.

Clearly, the goal of any procedure for the trace determination of selenium via the formation of hydrogen selenide is to maximize the amount of hydrogen selenide removed from the solution. Although many previous hydrogen selenide generation procedures, both batch and FI, have been optimized for just this situation, the approaches used have always been empirical. Reports of such studies contain little or no explicit discussion of the role of the factors which affect the distribution of hydrogen selenide gas between the aqueous and vapor phases. In an FI system, as opposed to a batch system, a description of this distribution is complicated further by kinetic effects. Kinetic effects would seem to be relevant in the case of the salting-out effect, as there appear to be no reports of enhanced signals for procedures based on batch generation and separation of the hydrogen selenide. The results obtained in the present study indicate that the effect is not observed when a batch reactor is used. Presumably the action of bubbling argon through the bulk liquid (combined with the evolution of hydrogen-see the following section) provides a mechanism for the removal of the same proportion of the analyte regardless of the dissolved salt content.

Previous work ${ }^{18}$ with this system showed that the overall efficiency for the transfer of selenium into the furnace by HG inatomizer trapping was $75 \%$. Sturgeon et al. ${ }^{19}$ found the processes to be $79 \%$ efficient. It is therefore feasible that, if the missing $20-25 \%$ of the selenium is not removed from the solution, the salting out of this fraction could account for an increase in signal of $25-33 \%$.

\section{Overcoming the effect}

As can be seen in Fig. 4, increasing the concentration of sodium borohydride increased the sensitivity and reduced the magnitude of the difference in signal between samples which contained salt and samples which did not. A concentration of $1.5 \% \mathrm{~m} / \mathrm{v}$ of sodium borohydride was sufficient to remove any significant differences between the signals for up to $20 \%$ of sodium chloride in solution. The use of sodium borohydride concentrations above this value resulted in violent reactions, which caused large amounts of solution to be transferred to the graphite tube, adversely affecting the results. As the concentra-

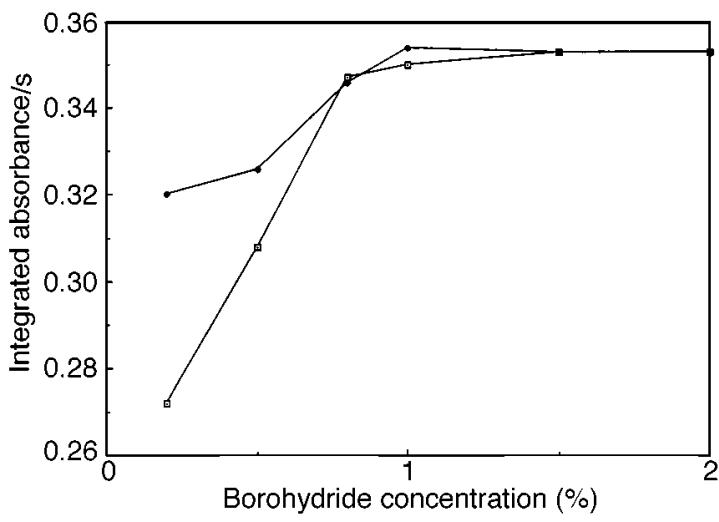

Fig. 4 Variation of signal for $5 \mathrm{ng} \mathrm{ml}^{-1}$ Se with different borohydride concentrations. $\square$, No salt in sample; $\diamond, 20 \%$ of salt in sample. 
tion of borohydride is increased, the distribution of the hydride between the two phases must be shifted towards the gas phase to such an extent that the salting-out process is insignificant. Therefore, one possible strategy for overcoming the salting-out effect would be the use of higher concentrations of borohydride. However, there are practical problems associated with excess foaming and bubble formation in the gas-liquid separator that may make this approach problematic in practice. In addition, interferences from transition metals are increased as the borohydride concentration is increased. However, the other two designs of gas-liquid separator were more tolerant to the use of increased borohydride concentrations and with $2.0 \%$ of borohydride gave signals which were around $94 \%$ of that of the PerkinElmer device operated with $0.2 \%$ of borohydride.

The addition of salt solutions through an extra line added to the manifold lowered the sensitivity. However, as can be seen in Fig. 5, the addition of salt to the borohydride solution produced an increase in sensitivity for solutions without salt such that there was no significant difference due to the presence of salt in the sample. The 'salting-out ability' (i.e., concentration, charge, size) of the salt in the reductant stream must be sufficiently high to eliminate any differences between samples and standards.

\section{Conclusion}

It is concluded that the presence of high salt concentrations produces a positive interference in the FI-HG-ETAAS determination of arsenic and selenium due to increased transfer of the hydride into the vapor phase. It is proposed that this is a thermodynamic rather than a kinetic effect, that is, the presence of the dissolved salt affects the equilibrium distribution of the hydrogen selenide or arsine between the gas and liquid phases. Although evidence was found for the transport of aerosol to the atomizer, this was not related to the signal enhancement. The enhancement effect could be compensated for by adding salt to the borohydride reagent or eliminated by increasing the borohydride concentration. This latter strategy was not suitable for the FI system used because the design of the gas-liquid separator was such that bulk liquid was carried to the atomizer,

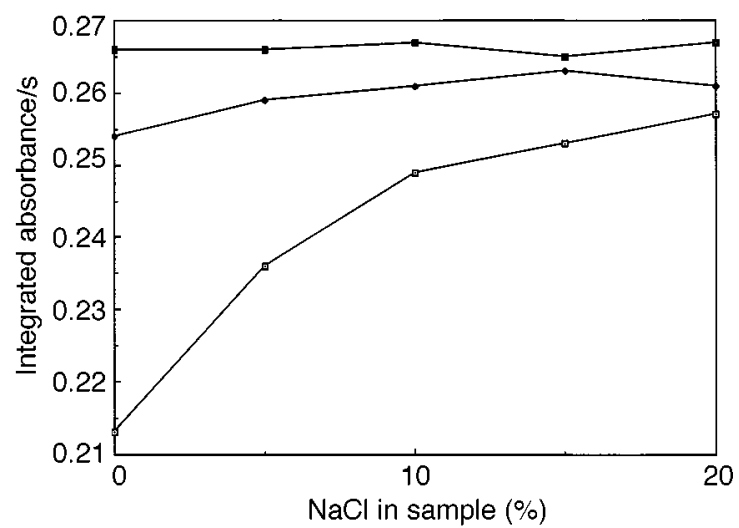

Fig. 5 Variation of signal for $5 \mathrm{ng} \mathrm{ml}^{-1} \mathrm{Se}$ for samples with different $\mathrm{NaCl}$ concentrations for different concentrations of $\mathrm{NaCl}$ in the borohydride solution. $\square, \mathrm{No} \mathrm{NaCl}$ in borohydride; $\diamond, 20 \%$ of $\mathrm{NaCl}$ in the borohydride solution; and $\mathbf{\square}, 40 \%$ of $\mathrm{NaCl}$ in the borohydride solution. adversely affecting the trapping ability of the furnace coating. For FI-HG with continuous atomization, the design of the gasliquid separator is governed by the need to minimize dispersion effects. As dispersion increases there is a consequent decrease in sensitivity and a decrease in throughput. However, when inatomizer trapping is used, the shape of the peak is, to a large extent, independent of the kinetics of any of the processes of generation and separation of the hydride. Hence the requirement for minimizing the dispersion processes in the gas-liquid separator could be relaxed and designs (such as those of the other two devices used in this study) which allowed a greater tolerance to borohydride concentration could be used. Several designs of gas-liquid separator have been evaluated. ${ }^{20,21}$

The authors thank Perkin-Elmer for financial support for Nils Sundin and Robert Ellis and the provision of equipment, and Walter Slavin, Bonaire Technologies, for many helpful discussions.

\section{References}

1 Matusiewicz, H., and Sturgeon, R. E., Spectrochim. Acta, Part B, 1996, 51, 377.

2 Fang, Z., Flow Injection Atomic Absorption Spectrometry, WileyInterscience, Chichester, 1996.

3 Carnrick, G., personal communication, 1995.

4 Sundin, N. G., Tyson, J. F., McIntosh, S. A., and Hanna, C. P., Spectrochim. Acta, Part B, 1995, 50, 369.

5 Perkin-Elmer, Manufacturer's Instruction Manual, Part Number B050-9907, Publication B3212.10, Perkin-Elmer, Norwalk, CT, 1993.

6 Hanna, C. P., Carnrick, G. R., McIntosh, S. A., Guyette, L. C., and Bergemann, D. E., At. Spectrosc., 1995, 16, 82.

7 Welz, B., and Schubert-Jacobs, M., J. Anal. At. Spectrom., 1986, 1, 23.

8 Tyson, J. F., Offley, S. G., Seare, N. J., Kibble, H. A. B., and Fellows, C., J. Anal. At. Spectrom., 1992, 7, 315.

9 Bax, D., Agterdenbos, J., Worrell, E., and Beneken Kolmer, J., Spectrochim. Acta, Part B, 1988, 43, 1349.

10 Wickstrom, T., Lund, W., and Bye, R., Analyst, 1996, 121, 201.

11 Thompson, M., Pahlavanpour, B., Walton, S., and Kirkbright, G. F., Analyst, 1978, 103, 568 .

12 Cresser, M., in Sample Introduction in Atomic Spectrometry, ed. Sneddon, J., Elsevier, Amsterdam, 1990, ch. 2.

13 Atkins P. W., Physical Chemistry, Freeman, New York, 3rd edn., 1986.

14 Dack, M. R. J., Techniques of Chemistry Vol VII-Solutions and Solubility, Part 1, Wiley, New York, 1986.

15 Battino, R., and Clever, H. L., Chem. Rev., 1966, 66, 395.

16 Glasstone, S., Physical Chemistry, Macmillan, London, 2nd edn., 1948.

17 Seto, Y., J. Chromatogr. A., 1994, 674, 25

18 Tyson, J. F., Sundin, N. G., Hanna, C. P., and McIntosh, S. A., Spectrochim. Acta, Part B, 1997, 52, 1773.

19 Sturgeon, R. E., Willie, S. N., Sproule, G. I., Robinson, P. T., and Berman, S. S., Spectrochim. Acta, Part B, 1989, 44, 667.

20 Hanna, C. P., Haigh, P. E., Tyson, J. F., and McIntosh, S., J. Anal. At. Spectrom., 1993, 8, 585.

21 Brindle, I. D., and Zheng, S., Spectrochim. Acta, Part B, 1996, 51, 1777.

Paper 8/02944J

Received April 20, 1998

Accepted June 9, 1998 